\title{
Uso de resonancia magnética con secuencia de difusión no-ecoplanar para la detección de colesteatoma en pacientes con cirugía de oído previa: Presentación de 4 casos
}

\section{Use of non-echo-planar diffusion-weighted magnetic resonance imaging for the detection of cholesteatoma in patients with previous ear surgery: Presentation of 4 cases}

\author{
Natalia Cabrera $\mathbf{S}^{1}$, Cecilia Sedano $\mathbf{M}^{2}$, Paul H. Délano $\mathbf{R}^{1}$, Andrés Alvo $\mathbf{V}^{1}$.
}

\begin{abstract}
RESUMEN
El diagnóstico de colesteatoma es fundamentalmente clínico, pudiendo ser complementado con imágenes. Últimamente, el uso de resonancia magnética con secuencia HASTE ha surgido como herramienta diagnóstica sensible y específica para colesteatoma, permitiendo diferenciar éste del tejido inflamatorio-fibrótico presente en pacientes que han sido sometidos a cirugía de oído previa. En este trabajo se presentan cuatro casos de pacientes con antecedente de cirugía de oído y sospecha de colesteatoma en el mismo oído operado, realizándose RM HASTE y cirugía posterior. Obtuvimos una alta correlación entre la imagen y resultado quirúrgico. Se requiere mayor número de pacientes para determinar la sensibilidad y especificidad en el diagnóstico de colesteatoma en pacientes con cirugía de oídos previa.

Palabras clave: Colesteatoma, tomografía computarizada oídos, RM HASTE, cirugía de revisión de oídos.
\end{abstract}

\begin{abstract}
The diagnosis of cholesteatoma is mainly established by clinical signs,and can be improved by radiological imaging. Recently, the use of magnetic resonance imaging (MRI) with HASTE sequence has emerged as a sensitive and specific diagnostic tool for cholesteatoma, which allows differentiating it from inflammatory-fibrotic tissue present in patients who have undergone previous surgery ear. Here, we present four cases of patients with a history of ear surgery and a suspected cholesteatoma in the same ear, performing HASTE MRI and subsequent surgery, presenting a high correlation between $M R I$ and surgical outcome. More patients are needed to determine the sensitivity and specificity in the diagnosis of cholesteatoma inpatients with previous ear surgery.

Key words: Cholesteatoma, computed tomography, HASTE MRI, second-look surgery, revision surgery.
\end{abstract}

Médico de Servicio de Otorrinolaringología, Hospital Clínico Universidad de Chile.

Médico-Cirujano, Universidad de los Andes.

Recibido el 5 de enero de 2015. Aceptado el 20 de enero de 2015. 


\section{INTRODUCCIÓN}

El colesteatoma es una lesión quística benigna de comportamiento agresivo, compuesta de epitelio escamoso estratificado queratinizado. Está constituido por una matriz epitelial rodeada por un estroma inflamatorio de grosor variable (perimatriz), relleno de detritus descamativo ${ }^{1}$. Según su origen pueden ser congénitos (epitelio remanente en oído medio durante el desarrollo fetal) o adquiridos, siendo estos últimos primarios (a partir de una retracción timpánica) o secundarios (perforación timpánica, fractura o iatrogénico $)^{2,3}$.

El colesteatoma puede producir una erosión progresiva de las estructuras óseas del oído medio (cadena osicular, canal del nervio facial, laberinto óseo, y base de cráneo), pudiendo causar complicaciones severas, como meningitis y absceso intracraneal ${ }^{4}$, e incluso podría recurrir tras su extracción (10\%-15\%), o persistir colesteatoma residual $(5 \%-63 \%)^{5,6}$.

El diagnóstico de colesteatoma adquirido es fundamentalmente clínico, al observar en la otoscopía la queratina descamada en relación a un bolsillo de retracción timpánica ${ }^{7}$. La tomografía computarizada (TC) de oídos con corte fino $(0,5 \mathrm{~mm})$ sin contraste es el examen de elección para el estudio con imagenes del paciente con diagnóstico de colesteatoma ${ }^{8}$, pero la opacidad en la TC es inespecífica para colesteatoma, y el diagnóstico se basa principalmente en hallazgos tales como erosión ósea en ciertas localizaciones (pared lateral del epitímpano 0 de la cadena osicular) y medialización de la cadena osicular ${ }^{7,8}$. En términos generales la TC permite sospechar el diagnóstico, evaluar complicaciones derivadas de la erosión ósea en la cadena osicular, tegmen tympani, canal semicircular lateral o canal de Falopio, determinar extensión de la enfermedad y evaluar la anatomía del oído medio y de la cavidad mastoidea, contribuyendo principalmente en la planificación del procedimiento quirúrgico $0^{9,10}$.

Múltiples estudios han estimado el valor de la TC de oídos en el diagnóstico de colesteatoma al comparar sus resultados con los hallazgos quirúrgicos, encontrando una sensibilidad de $65 \%$ $91,9 \%$ y una especificidad de $55,6 \%-94,7 \%{ }^{11-14}$.

Debido a que la TC resulta inespecífica en algunas situaciones, tales como localizaciones atípicas en el oído medio, colesteatomas congénitos, y cavidades posquirúrgicas ${ }^{7}$, 0 en el diagnóstico diferencial de las masas de oído medio, como tejido de granulación, granuloma de colesterol, fibrosis posquirúrgica 0 encefalocele ${ }^{15}$, es que el uso de la resonancia magnética (RM) se ha convertido en una herramienta útil debido a que caracteriza de mejor manera los tejidos blandos.

Dentro de las diferentes técnicas utilizadas en RM, las secuencias de difusión molecular son las más utilizadas en el estudio del colesteatoma, y se basan en la restricción del movimiento de las moléculas de agua, como lo que se produce en este tipo de tumores, ricos en queratina, produciendo una señal hiperintensa; a diferencia de otros tejidos, como el tejido de granulación, donde las moléculas de agua tienen mayor movimiento, por lo que aparecen menos intensas ${ }^{16}$.

Las imágenes ecoplanares convencionales han sido desplazadas por las técnicas no-ecoplanares en el estudio del hueso temporal, debido a que estas secuencias tienen menos artefactos y permiten cortes más finos, permitiendo la detección de colesteatomas desde 2 milímetros ${ }^{17,18}$. Dentro de éstas, la más utilizada es la secuencia conocida como Half-Fourier acquired single-shot turbo spin echo (HASTE).

Cabe destacar la posible presencia de falsos negativos en casos de artefactos de movimiento y colesteatomas autoevacuados ${ }^{19}$, y falsos positivos en presencia de infección aguda de oído medio, polvo de hueso, láminas de silastic, quistes de retención mucosos, incluso en algunos casos de tejido de granulación, tejido cicatricial y cerumen ${ }^{17,18,20,21}$. Hay otras causas infrecuentes de falsos positivos tales como el teratoma de oído medio; recientemente se reportó un caso en nuestro servicio que simulaba clínicamente un colesteatoma congénito, y que presentó una RM HASTE positiva, pero con un patrón granular ${ }^{15}$.

Últimamente, se han publicado varios estudios con secuencias de difusión no-ecoplanar en el estudio de colesteatomas, incluyendo secuencias HASTE, mostrando excelente sensibilidad y especificidad en su detección, en especial en casos de sospecha de colesteatoma congénito, duda diagnóstica en la TC en casos de colesteatoma adquirido, y en la diferenciación con los cambios posquirúrgicos, en especial después de técnicas canal wall-up, lo que permitiría evitar el secondlook quirúrgico para la búsqueda de enfermedad residual o recurrente $e^{7,22}$. 
A continuación, presentamos cuatro casos clínicos de pacientes atendidos en el Hospital Clínico de la Universidad de Chile, con antecedente de cirugía de oído y posterior sospecha clínica de colesteatoma en el mismo oído operado, a quienes se les realizó RM HASTE, y cirugía posterior.

\section{CASOS CLÍNICOS}

\section{Caso 1}

Mujer de 47 años, con antecedente de timpanoplastía del oído izquierdo en 1985 y aticotomía del oído izquierdo en 2008, quien consultó por otorrea de oído izquierdo. En la otoscopía destacaba el injerto, probablemente pericondrio, con presencia de una dehiscencia anterior y retención de piel hacia ático. La RM HASTE, en corte axial, fue catalogada como dudosa para colesteatoma (Figura 1). Los hallazgos intraoperatorios y biopsia diferida fueron compatibles con colesteatoma en techo del antro mastoídeo.

\section{Caso 2}

Hombre de 18 años, con antecedente de cirugía radical de oído derecho por colesteatoma en 2012. Consulta en noviembre de 2013 por otorrea de oído derecho, presentando en la otoscopía aumento de volumen perlado en techo del antro mastoideo. La tomografía computarizada de oídos muestra oído derecho con cavidad radical amplia con tejido isodenso en antro mastoideo derecho. Ante duda diagnóstica se realizó RM HASTE la cual resultó positiva para colesteatoma en oído derecho (Figura 2). Los hallazgos intraoperatorios y biopsia diferida fueron compatibles con colesteatoma en techo timpánico, en especial hacia epitímpano anterior.

\section{Caso 3}

Niño de 12 años con antecedente de timpanoplastía de oído izquierdo con cartílago en 2011. Durante los controles se evidenció una lateralización del injerto con perforación posterior marginal pequeña y presencia de otorrea. Se realizó RM HASTE que resultó positiva para colesteatoma en epitímpano y mesotímpano de oído izquierdo (Figura 3). Los hallazgos intraoperatorios y biopsia diferida fueron compatibles con colesteatoma en epitímpano.

\section{Caso 4}

Mujer de 23 años, con antecedente de timpanoplastía bilateral en 2004. Durante los controles se evidenció en el oído derecho una retracción del hemitímpano anterior asociado a bolsillo sin fondo visible y granuloma timpánico que fue cauterizado, con posterior reducción de su tamaño. Se realizó RM HASTE, la cual resultó negativa para colesteatoma (Figura 4). Los hallazgos intraoperatorios y biopsia diferida no mostraron evidencia de colesteatoma, por lo que se realizó una timpanoplastía secundaria.

\section{DISCUSIÓN}

En los últimos años, el uso de resonancia magnética con secuencia HASTE ha surgido como una herramienta diagnóstica sensible (82\%-100\%) y específica (87\%-100\%) para colesteatoma ${ }^{7,23-25}$, permitiendo incluso diferenciar el colesteatoma del tejido inflamatorio-fibrótico presente en pacientes que han sido sometidos a cirugía de oído previa, lo cual podría ayudar a identificar los pacientes que se beneficiarán de una cirugía de revisión ${ }^{25,26}$. Se han utilizado otras secuencias de RM, como T1 con realce tardío con gadolinio, presentando menor sensibilidad y especificidad con respecto a la secuencia de difusión no-ecoplanar ${ }^{27}$. Esta última ha demostrado su utilidad en el diagnóstico de colesteatoma residual o recurrente de hasta $2 \mathrm{~mm}$, por lo que cabe destacar que un examen negativo no descarta la enfermedad ${ }^{16,28,29}$. Más aún, Alvo y cols, recientemente, han demostrado una alta correlación entre los hallazgos de la RM HASTE y los quirúrgicos, en pacientes con bolsillos de retracción y sospecha de colesteatoma, encontrando una sensibilidad de $90 \%$ y especificidad de $100 \%{ }^{30}$.

Majithia y cols demostraron que existe una buena correlación entre la etapificación de la extensión del colesteatoma primario observada en la RM HASTE en comparación con los hallazgos quirúrgicos, en el contexto de una imagen de oído medio completamente velado en la TC de oído ${ }^{31}$. 


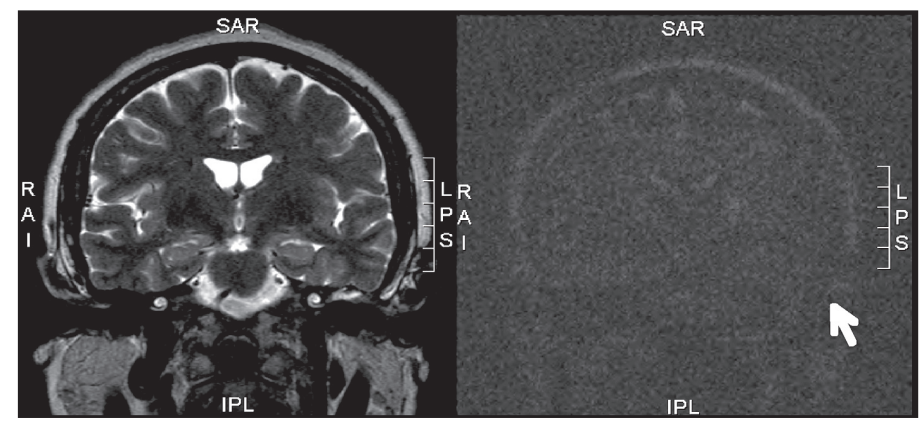

Figura 1. RM oídos secuencia T2 (izq) y HASTE (der), corte coronal, con resultado dudoso (flecha) para colesteatoma en oído izquierdo.

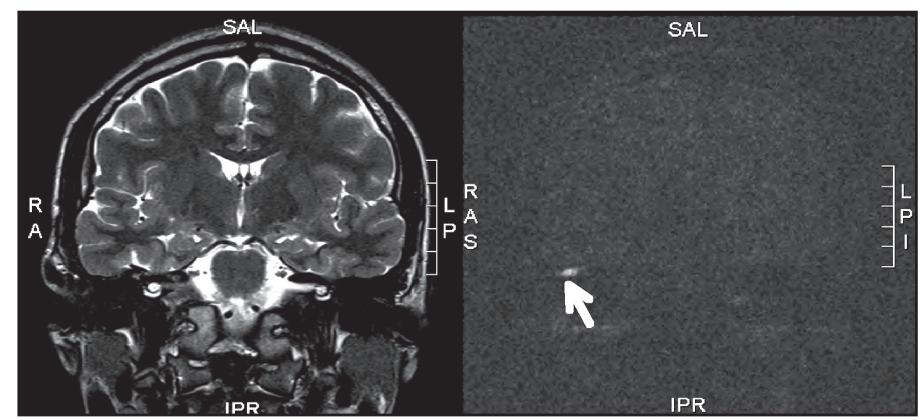

Figura 2. RM oídos secuencia T2 (izq) y HASTE (der), corte coronal, con resultado positivo para colesteatoma en oído derecho (flecha).

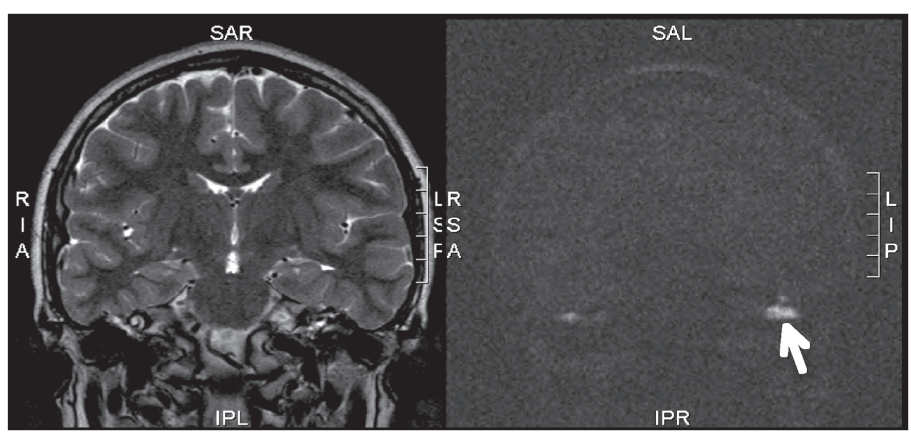

Figura 3. RM oídos secuencia T2 (izq) y HASTE (der), corte coronal, con resultado positivo para colesteatoma en oído izquierdo (flecha).

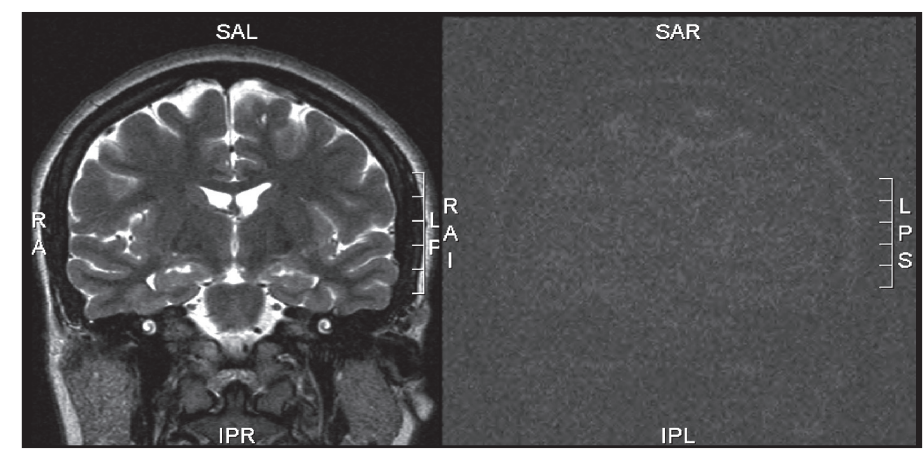

Figura 4. RM oídos secuencia T2 (izq) y HASTE (der), corte coronal, con resultado negativo para colesteatoma en oído derecho. 
En cuanto al diagnóstico de colesteatoma residual o recurrente, se ha visto que el diagnóstico clínico puede ser menos confiable, por lo que las imágenes son actualmente prioritarias antes de realizar una cirugía de revisión, siendo de elección la RM con secuencia HASTE ${ }^{32,33}$. Khemani y cols evaluaron la utilidad de la RM HASTE en la detección, localización y predicción de la extensión del colesteatoma posoperatorio, concluyendo que, si bien tiene buena sensibilidad (S) y especificidad (E) en la detección (S 82\%, E 90\%) y localización (S 75-88\%, E 94-100\%), podría subestimar el tamaño real del colesteatoma e incluso no detectar lesiones pequeñas $(<2 \text { milímetros })^{34}$.

La discusión de nuestros casos nos lleva a sugerir que el diagnóstico de colesteatoma es principalmente clínico, pero en casos de mayor complejidad, como son los pacientes con antecedente de cirugía de oído, la RM HASTE es una herramienta sensible y específica para el diagnóstico de colesteatoma. En el caso 2 se muestra un caso en el cual la sospecha clínica no se aclaró con la TC, por lo que la RM HASTE fue esencial para decidir reoperar al paciente. En el caso 3, se muestra un paciente pediátrico con antecedente de timpanoplastía, que presentó lateralización del injerto y otorrea, lo cual hizo sospechar un posible colesteatoma, por lo que la RM HASTE fue de gran utilidad al apoyar el diagnóstico de colesteatoma, lo que fue ratificado en la cirugía y biopsia. En el caso 4 no existía sospecha clínica directa de colesteatoma, pero ante una duda razonable debido a la presencia de un bolsillo de retracción sin fondo visible, se realizó la RM HASTE, la cual confirmó la sospecha clínica de ausencia de colesteatoma.

$\mathrm{Si}$ bien, en esta serie de casos obtuvimos una buena correlación entre la imagen y resultado quirúrgico $(75 \%)$, se requiere mayor número de pacientes para determinar la sensibilidad y especificidad en el diagnóstico de colesteatoma posquirúrgico.

\section{CONCLUSIÓN}

La RM HASTE es actualmente una herramienta de gran utilidad en el diagnóstico de colesteatoma residual o recurrente en pacientes con antecedentes de cirugía previa de oído medio, lo que permitiría evitar la cirugía de revisión en algunos casos. Se debe tener precaución en pacientes con RM negativa, ya que tumores muy pequeños, de menos de 2 milímetros, podrían no ser detectados, por lo que se debe continuar el seguimiento clínico, y eventualmente radiológico de estos pacientes. También se debe tener en cuenta que existen situaciones que podrían producir falsos positivos en la RM, como por ejemplo las infecciones de oído medio, por lo que se debe tener cautela en la interpretación de los hallazgos y siempre correlacionar con la clínica. Para concluir, actualmente, la RM HASTE es un método no invasivo confiable para detectar el colesteatoma residual o recurrente, lo cual podría reemplazar a la cirugía de revisión.

\section{Agradecimientos}

Este trabajo fue financiado por el concurso de Investigación de la Sociedad Chilena de Otorrinolaringología SOCHIORL otorgado el año 2011 (Dr. Andrés Alvo).

\section{BIBLIOGRAFÍA}

1. Semaan M, Megerian, CA. The pathophysiology of cholesteatoma. Otolaryngol Clin North Am 2006; 39(6): 1143-59.

2. Nevoux J, Lenoir, M, Roger, G, et Al. Childhood cholesteatoma. Ann Otorhinolaryngol Head Neck Dis 2010; 127: 143-50.

3. Chang P, KIm, S. Cholesteatoma: diagnosing the unsafe ear. Aust Fam Physician 2008; 37: 631-8.

4. Wu J, Jin, Z, YANG, JM, et AL. Extracranial and intracranial complications of otitis media: 22-year clinical experience and analysis. Acta Otolaryngol 2012; 132: 261-5.

5. Stott C, Royer, M, Ortúzar, L, Pavón, M Resultados auditivos posatico antrostomía con antroexclusión. Rev Otorrinolaringol Cir Cabeza Cuello 2007; 67: 129-34.

6. LIN J, OghaLAl, J. Can Radiologic Imaging Replace Second-Look Procedures for Cholesteatoma? Laryngoscope 2010; 121: 4-5.

7. Más-Estellés F, Mateos-Fernández, M, CrrascosaBisquert, B, Facal de Castro, F, Puchades-Román, I, Morera-Pérez, C. Contemporary non-echo- 
planar diffusion-weighted imaging of middle ear cholesteatomas. Radiographics 2012; 32(4): 1197-213.

8. Alzérreca e GC, Zamorano R, Salas A, Délano P. Resonancia magnética cerebral con secuencia difusión - HASTE en la evaluación clínica del colesteatoma. Rev Otorrinolaringol Cir Cabeza Cuello 2011; 71(3): 249-56.

9. Ayache D, Darrouzet, V, Dubrulle, F, Vincent, C, Bobin, S, Williams, M, Martin, C. Imaging of non-operated cholesteatoma: Clinical practice guidelines. European Annals of Otorhinolaryngology, Head and Neck diseases 2012; 129: 148-52.

10. Ganaha A. Efficacy of diffusion-weighted magnetic resonance imaging in the diagnosis of middle ear cholesteatoma. Auris Nasus Larynx 2011; 38(3): 329-34.

11. Yildirim-Baylan M, Ozmen, CA, Gun, R, Yorgancilar, E, AкKu, Z, TopCu, I. An evaluation of preoperative computed tomography on patients with chronic otitis media. Indian J Otolaryngol Head Neck Surg 2012; 64(1): 67-70.

12. Tatlipinar A, Tuncel, A, Öredik, EA, Gökçeer, T, Uslu, C. The role of computed tomography scanning in chronic otitis media. Eur Arch Otorhinolaryngol 2012; 269(2): 33-8.

13. Gerami H, Naghavi, E, Wahabi-Moghadam, M, Forghanparast, K, Akbar, MH. Comparison of preoperative computerized tomography scan imaging of temporal bone with the intra-operative findings in patients undergoing mastoidectomy. Saudi Med J 2009; 30(1): 104-8.

14. Payal G, Pranjal, K, Gul, M, Mittal, MK, Ral, AK. Computed tomography in chronic suppurative otitis media: value in surgical planning. Indian J Otolaryngol Head Neck Surg 2012; 64(3): 2259.

15. León F, Alvo, A, Sanhueza, D, Délano, P, Stott, C. HASTE Diffusion-Weighted Magnetic Resonance Imaging of Middle Ear Teratoma. Otol Neurotol 2014.

16. Jindal M, Doshi, J, SRivastav, M, Wilcock, D, Irving, $\mathrm{R}, \mathrm{DE}, \mathrm{R}$. Diffusion-weighted magnetic resonance imaging in the management of cholesteatoma. Eur Arch Otorhinolaryngol 2010; 267(2): 1815.

17. De Foer B, Vercruysse, JP, Spaepen, M, Somers, T, Pouillon, M, Offeciers, E, Casselman, JW.
Diffusion-weighted magnetic resonance imaging of the temporal bone. Neuroradiology 2010; 52 : 785-807.

18. Moura M, Taranto, D, García, M. Cholesteatoma: utility of non-echo-planar diffusion-weighted imaging. Radiol Bras 2012; 45(5): 283-7.

19. Baráth K, Huber, AM, Stämpfl, P, Varga, Z, Kollias, S. Neuroradiology of cholesteatomas. AJNR Am J Neuroradiol 2011; 32(2): 221-9.

20. Karandikar A, Goh, J, Loke, S, Yeo, S, Tan, T. Mucous retention cyst of temporal bone: a mimic of cholesteatoma on DW-MRI. Am J Otolaryngol 2013; 34(6): 753-4.

21. Profant M, Sláviková, K, Kabátová, Z, Slezák, P, Waczulíková, I. Predictive validity of MRI in detecting and following cholesteatoma. Eur Arch Otorhinolaryngol 2012; 269(3): 757-65.

22. Schwartz K, Lane, JI, Bolster, BD Jr, Neff, $B A$. The utility of diffusion-weighted imaging for cholesteatoma evaluation. AJNR Am J Neuroradiol 2011; 32(3): 430-6.

23. Sharifian H. Diagnostic accuracy of non-echoplanar diffusion-weighted MRI versus other MRI sequences in cholesteatoma. J Med Imaging Radiat Oncol 2012; 56(4): 398-408.

24. Plouin-Gaudon I, Bossard, D, Ayari-Khalfallah, S, Froehlich, P. Fusion of MRIs and CT scans for surgical treatment of cholesteatoma of the middle ear in children. Arch Otolaryngol Head Neck Surg 2010; 136(9): 878-83.

25. Li P, Linos, E, Gurgel, R, Fischbein, N, Blevins, N. Evaluating the Utility of Non-Echo Planar Diffusion-Weighted Imaging in the Preoperative Evaluation Of Cholesteatoma: A Meta-analysis. Laryngoscope 2013; 123(5): 1247-50.

26. Ilica A, Hidir, Y, Bulakba I, N, Satar, B, Güvenç, I, Arslan, HH, ImRE, N. HASTE diffusion-weighted MRI for the reliable detection of cholesteatoma. Diagn Interv Radiol 2012; 18(2): 153-8.

27. De Foer B, Vercruysse, JP, Bernaerts, A, Meerschaert, J, Kenis, C, Pouillon, M, De Beuckeleer, L, Michiels, J, Bogaerts, K, Deckers, F, Somers, T, Hermans, R, Offeciers, E, Casselman, J. Non-Echo-planar Diffusion-weghted MR Imaging versus Delayed Gadolinium-enhanced T1-weighted MR Imaging - Value in Detection. Radiology 2010; 255(3): FALTA AÑO.

28. Aikele P, Kittner, T, Offergeld, C, Kaftan, H, HütTenbrink, K, Laniado, M. Diffusion-Weighted 
MR Imaging of Cholesteatoma in Pediatric and Adult Patients Who Have Undergone Middle Ear Surgery. AJR 2003; 181.

29. Vercruysse J, De Foer, B, Puillon, M, Somers, T, CAsselman, J, OfFeciers, E. The value of diffusionweighted MR imaging in the diagnosis of primary acquired and residual cholesteatoma: a surgical verified study of 100 patients. Eur Radiol 2006; 16: 1461-7.

30. Alvo A, Garrido, C, Salas, A, Miranda, G, Stott, C, Délano, P. Use of Non-Echo-Planar Diffusion Weighted MI Imaging for the Detection of Cholesteatomas in High-Risk Tympanic Retraction POckets. AJNR Am J Neuroradiol 2014; 35: 1-5.

31. Majithia A, Lingam, R, Nash, R, Khemani, S, Kalan, A, Singh, A. Staging primary middle ear cholesteatoma with non-echoplanar (half-
Fourier-acquisition single-shot turbo-spinecho) diffusion-weighted magnetic resonance imaging helps plan surgery in 22 patients: our experience. Clin Otolaryngol2012; 37(4): 325-30.

32. Khemani S, Singh, A, Lingam, R, Kalan, A. Imaging of postoperative middle ear cholesteatoma. Clin Radiol 2011; 66(8): 760-7.

33. Cagatay N, Cimsit, C, Baysal, B, cagatay, I, Ozbilgen $S$, Ayanoglu, E. Diffusion-weighted MR imaging in postoperative follow-up:Realiability for detection of recurrent cholestetatoma. European Journal of Radiology 2010; 74: 121-3.

34. Khemani S, Lingman, R, Kalan, A, Singh, A. The value of non-echo planar HASTE diffusion-weighted MR imaging in the detection, localisation and prediction of extent of postoperative cholesteatoma. Clin Otolaryngol 2011; 36: 306-12. 